\title{
Antibody profile to Borrelia burgdorferi in veterinarians from Nuevo León, Mexico, a non-endemic area of this zoonosis
}

\author{
Cassandra M. Skinner-Taylor ${ }^{1}$, Maria S. Flores ${ }^{1}$, José A. Salinas², Katiushka Arevalo-Niño,

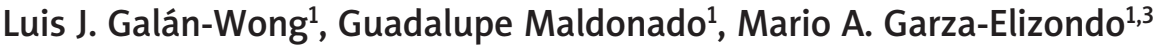 \\ ${ }^{1}$ Universidad Autonóma de Nuevo León, School of Biological Sciences, Institute of Biotechnology, Nuevo León, Mexico \\ ${ }^{2}$ Universidad Autonóma de Nuevo León, Faculty of Veterinary Medicine and Zootechnics, Nuevo León, Mexico \\ ${ }^{3}$ Rheumatology Unit, Medical Faculty and University Hospital Dr. José Eleuterio González, Nuevo León, Mexico
}

\begin{abstract}
Objectives: Lyme disease is a tick-borne disease caused by infections with Borrelia. Persons infected with Borrelia can be asymptomatic or can develop disseminated disease. Diagnosis and recognition of groups at risk of infection with Borrelia burgdorferi is of great interest to contemporary rheumatology. There are a few reports about Borrelia infection in Mexico, including lymphocytoma cases positive to $B$. burgdorferi sensu stricto by PCR and a patient with acrodermatitis chronica atrophicans. Veterinarians have an occupational risk due to high rates of tick contact.

The aim of this work was to investigate antibodies to Borrelia in students at the Faculty of Veterinary Medicine and Zootechnics, at Nuevo León, Mexico, and determine the antibody profile to B. burgdorferi antigens.

Material and methods: Sera were screened using a C6 ELISA, IgG and IgM ELISA using recombinant proteins from B. burgdorferi, B. garinii and B. afzelii. Sera with positive or grey-zone values were tested by IgG Western blot to $B$. burgdorferi sensu stricto.

Results: All volunteers reported tick exposures and $72.5 \%$ remembered tick bites. Only nine persons described mild Lyme disease related symptoms, including headaches, paresthesias, myalgias and arthralgias. None of the volunteers reported erythema migrans. Nine samples were confirmed by IgG Western blot. The profile showed $89 \%$ reactivity to OspA, $67 \%$ to p 83 , and $45 \%$ to BmpA.

Conclusions: Positive sera samples shared antibody reactivity to the markers of late immune response 83 and BmpA, even if individuals did not present symptoms of Lyme arthritis or post-Lyme disease. The best criterion to diagnose Lyme disease in our country remains to be established, because it is probable that different strains coexist in Mexico. This is the first report of antibodies to B. burgdorferi in Latin American veterinarians. Veterinarians and high-risk people should be alert to take precautionary measures to prevent tick-borne diseases.
\end{abstract}

Key words: antibodies, Lyme disease, B. burgdorferi, veterinarians, ticks, OspA.

\section{Introduction}

Lyme disease is an infection caused by the spirochete Borrelia burgdorferi following transmission to humans by the bite of infected Ixodes ticks. In North America, Lyme disease is caused by B. burgdorferi sensu stricto. In contrast to North America, in Europe the infection is caused by a greater diversity of species: B. Burgdorferi sensu stricto, B. garinii, or B. afzelii $[1,2]$. Persons infected with Borrelia can be asymptomatic or can develop disseminated disease [3]. Borrelia burgdorferi may induce a wide range of clinical symptoms that affect the musculoskeletal apparatus, the nervous system, heart and

Address for correspondence:

Maria S. Flores, Pedro de Alba y Barragán s/n. Ciudad Universitaria, 66451 San Nicolás de los Garza, Nuevo León, Mexico,

e-mail: floresgms@yahoo.com

Submitted: 4.05.2016, Accepted: 30.06.2016 
skin. Lyme disease may be localized, such as in erythema migrans or disseminated [4]. About $10 \%$ of patients with Lyme disease continue to experience musculoskeletal pain and cognitive dysfunction after recommended antibiotic treatment. This condition is called post-Lyme disease syndrome (PLDS) [5].

Due to limitations in direct detection of $B$. burgdorferi sensu lato in clinical specimens, antibody detection supports a clinical diagnosis of Lyme disease. Antigenic composition variations exist among different commercial kits. Whether B. burgdorferi sensu lato antigens elicit IgM versus IgG antibodies depends on the duration of infection and the manifestation of the disease [4].

The highest incidence of Lyme disease in the USA occurs in the northeast, Mid-Atlantic States and upper Midwest. Other areas are considered non-endemic, such as the southwest states. For example, Texas has been considered a zoonotically non-endemic area; however, Harvey and Salvato [6] found among chronically ill patients in Houston large numbers with B. burgdorferi positive PCR. Borrelia burgdorferi has been isolated from Texas ticks and a variety of household and wild animals [7-9]. The Texas Veterinary Medical Diagnostic Laboratory reported $5.5 \%$ of 2,409 canine serum samples with antibodies to B. burgdorferi [10]. Although dogs cannot transmit Borrelia directly to humans, a canine seroprevalence of > 5\% may be a marker for increased risk of human Lyme disease [11].

Even though Mexico has a great variety of tick species, some of which are in the genus Ixodes [12], there are few reports about Borrelia infection in Mexico [13] Galaviz-Silva et al. [14] reported that $1.56 \%$ of ticks removed from dogs were positive for B. burgdorferi by PCR. The positive ticks were identified as $D$. variabilis. They found $0.9 \%$ of tested dog blood samples positive to B. burgdorferi OspA. Antibodies to Borrelia have been detected in horses and dogs, as well as B. burgdorferi DNA in a human skin biopsy and dog synovial fluids [15-17]. Gordillo et al. found 3 to $6 \%$ seroprevalence in a frozen sera bank from Mexico City and northeast Mexico. The same group identified two lymphocytoma cases, positive to $B$. burgdorferi sensu stricto by PCR. The sera from both patients were positive by Western blot [18, 19]. Feria-Arroyo et al. [20] detected B. burgdorferi from I. scapularis ticks collected in the south Texas-Mexico transboundary region, and they proposed that climate change might affect the suitability of habitats for ticks.

Zoonotic pathogens are responsible for many human disease threats; consequently, people who are exposed to ticks have an increased risk of contracting Lyme disease. Veterinarians are at risk associated with their jobs [21, 22]. The aim of this study was to investigate antibodies to $B$. burgdorferi in order to determine whether students and teachers at the Faculty of Veterinary Medicine and Zootechnics, from Nuevo León, Mexico, had been exposed to this pathogen and determine the antibody reactivity profiles using the IgG Western blot test.

\section{Material and methods \\ Study population}

Volunteers $(n=40)$ (21 female/19 male) average age 23 years (range 18-45 years) were enrolled from students at the Faculty of Veterinary Medicine and Zootechnics, UANL, at Nuevo León, Mexico, a border state to the United States located in northeast Mexico. All participants were living in the Monterrey metropolitan area except one veterinarian student living at a rural location. Nobody had traveled to areas of high endemicity. Participants provided informed consent, and a survey was carried out to assess their risk of tick exposure, tick bite history, development of erythema migrans, or the presentation of clinical symptoms suggestive of Lyme disease. The Research and Ethics Committee of the Facultad de Ciencias Biológicas UANL approved the research protocol, as well as the Research and Ethics Committee of the University Hospital José Eleuterio Gonzalez, UANL, at Monterrey Nuevo León, Mexico (Register number RE06-017). The research was carried out according to the World Medical Association Declaration of Helsinki of 1975, revised 1983.

\section{Diagnostic tests}

Serum from respective participants was screened using a C6 ELISA, using the antigen derived from the Borrelia spp. immunodominant protein VIsE (C6 ELISA, Immunetics, Boston, MA, USA); IgG ELISA BORG0040 and IgM ELISA BORM0040 (NovaLisa NovaTec, Immunodiagnostica, GmbH, Dietzenbach, Germany). NovaLisa Borrelia burgdorferi IgG-ELISA contains the recombinant epitope OspC of B31 (B. burgdorferi sensu stricto), 20047 and T25 (B. garinii), p100, and p18 of PKo (B. afzelii), and p41i of PBi (B. garinii). Manufacturers offer $98.8 \%$ sensitivity, $100 \%$ specificity. The Nova Lisa Borrelia burgdorferi IgM-ELISA contains the recombinant epitope OspC of PKo (B. afzelii) and 20047 (B. garinii) and p41i of PBi (B. garinii). Manufacturers offer $93 \%$ sensitivity, $98.8 \%$ specificity. Manufacturers advertise the inclusion of recombinant proteins to avoid extensive cross-reactions with antibodies to Treponema pallidum, Leptospira and Borrelia recurrentis.

Treponema pallidum ELISA (Abbott Murex, Wiesbaden, Germany) was performed to rule out cross-reactions with Treponema spirochete.

Based on a two-tier-testing protocol, positive or grey zone ELISA specimens were confirmed by IgG We- 
stern blot analysis, designed to probe B. burgdorferi sensu stricto antigens (Euroimmun, Lübeck, Germany). The Western blot kits used in this study did not include VIsE. Each strip was scanned, compared to the positive control, and automatically evaluated using the EURO blot Scanner that carries out automatic identification, quantification, and assignment of bands, according to a lot-specific evaluation matrix provided by Euroimmun. Each kit contained a membrane strip of the same lot incubated with a positive reference serum (Euroimmun, Lübeck, Germany). Positive Western blots were defined according to the manufacturer's instructions: positive reactivity to more than one $B$. burgdorferi specific band of $18 \mathrm{kDa}, 21 / 22 \mathrm{kDa}, 25 \mathrm{kDa}, 31 \mathrm{kDa}, 34 \mathrm{kDa}, 39 \mathrm{kDa}$, or $83 \mathrm{kDa}$ antigens plus several bands of $17 \mathrm{kDa}, 28 \mathrm{kDa}$, 32 kDa, 36 kDa, 43 kDa, 47 kDa, 50 kDa, 57 kDa, 59 kDa, $62 \mathrm{kDa}$, and $75 \mathrm{kDa}$ or a $41 \mathrm{kDa}$ antigen. Indeterminate results obtained with reactivity to unspecific bands were considered negative for the purposes of this study.

\section{Results}

Nineteen samples (47.5\%) were positive or registered within the grey zone by at least one ELISA test. As expected, IgM ELISA assays resulted in more positive responses than the IgG and C6 ELISA tests (Fig. 1).

All volunteers reported tick exposures and 29 (72.5\%) recall being bitten by ticks. Only nine persons (22.5\%) described mild Lyme disease related symptoms, including headaches $(67 \%)$, paresthesias (33\%), myalgias (56\%), and arthralgias (44\%). None of the volunteers remembered erythema migrans.

Only IgG Western blot was tested to avoid potential cross-reactivity by IgM Western blot. Only positive or equivocal ELISA specimens in any of the three tests C6 ELISA, IgG ELISA or IgM ELISA were confirmed by IgG Western blot analysis designed to analyze antibodies to

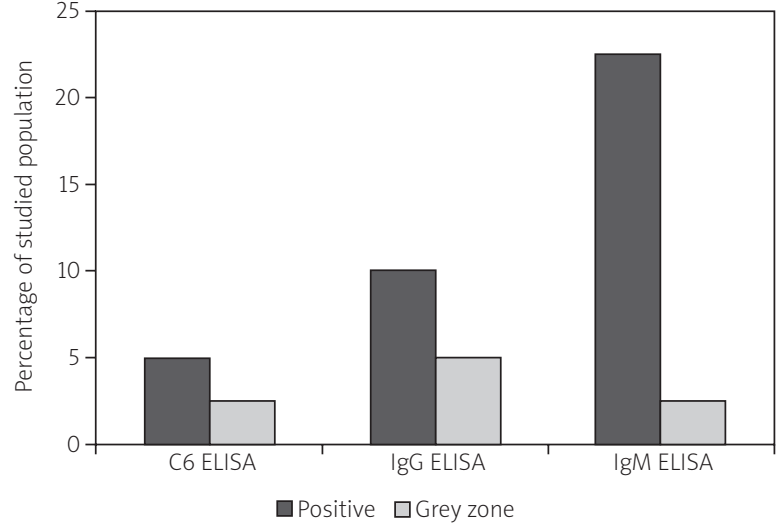

Fig. 1. Distribution of samples that gave positive or grey zone ELISA values. IgM, IgG or C6 ELISA tests $(n=40)$.

$B$. burgdorferi sensu stricto antigens. Indeterminate results obtained with reactivity to unspecific bands were considered negative for the purposes of this study. We tested by Western blot the IgG ELISA specimens in the grey zone because they presented values at the borderline, but we did not test specimens that gave negative results in the three ELISA tests. The IgM ELISA negative samples tested by Western blot were positive by $\mathrm{C} 6$ ELISA or IgG ELISA.

Western blots to identify IgG antibodies to B. burgdorferi detected nine positive samples. None of these samples was positive in the three ELISA tests employed. All positive individuals reported tick exposures and five recalled being bitten by ticks. Two samples with results giving IgG ELISA in the cut-off value gave IgG Western blot positive. Only one specimen that tested positive using the C6 ELISA was also positive when screened using the IgG Western blot (Table I). Another sample with a C6 positive score gave an indeterminate result

Table I. Characteristics of participants with antibodies to B. burgdorferi

\begin{tabular}{|c|c|c|c|c|c|c|c|c|}
\hline $\begin{array}{l}\text { Age } \\
\text { years }\end{array}$ & Gender & $\begin{array}{c}\text { Tick } \\
\text { exposure }\end{array}$ & Tick bite & $\begin{array}{l}\text { Lyme disease } \\
\text { symptoms }\end{array}$ & ELISA C6 & ELISA IgM & ELISA IgG & $\begin{array}{l}\text { IgG Western } \\
\text { blot }\end{array}$ \\
\hline 22 & M & + & - & - & - & + & - & + \\
\hline 21 & $M$ & + & - & - & - & - & + & + \\
\hline 21 & W & + & + & - & - & + & - & + \\
\hline 21 & W & + & + & + & + & - & - & + \\
\hline 22 & $M$ & + & + & - & - & - & + & + \\
\hline 45 & $M$ & + & + & - & - & - & $+/-$ & + \\
\hline 22 & $M$ & + & - & - & - & + & - & + \\
\hline 22 & W & + & - & - & - & - & $+/-$ & + \\
\hline 22 & W & + & + & - & - & + & - & + \\
\hline
\end{tabular}

M-man; W-woman; + positive; - negative; +1-Grey zone or borderline 
Table II. Antibody reactivity to B. burgdorferi antigens by IgG Western blot

\begin{tabular}{|c|c|c|c|c|c|c|c|c|c|c|c|c|c|c|c|c|c|c|}
\hline \multirow{2}{*}{$\begin{array}{l}\text { Sam- } \\
\text { ples }\end{array}$} & \multicolumn{11}{|c|}{ Non-specific antigens } & \multicolumn{7}{|c|}{ Borrelia burgdorferi specific antigens } \\
\hline & P17 & P28 & P32 & P36 & P43 & P47 & P50 & P57/59 & P62 & P75 & $\begin{array}{l}\text { Flag } \\
\text { P41 }\end{array}$ & P18 & P21/22 & $\begin{array}{l}\text { OspC } \\
\text { P25 }\end{array}$ & $\begin{array}{c}\text { OspA } \\
\text { P31 }\end{array}$ & $\begin{array}{c}\text { OspB } \\
\text { P34 }\end{array}$ & $\begin{array}{c}\text { Bmp } \\
\text { A P39 }\end{array}$ & P83 \\
\hline 1 & - & - & - & - & - & - & - & + & + & + & + & - & - & + & + & - & + & + \\
\hline 2 & - & - & - & - & - & - & - & + & + & + & + & - & - & - & + & - & - & + \\
\hline 3 & + & + & - & + & - & - & - & + & + & + & + & & - & - & + & - & - & + \\
\hline 4 & - & - & - & - & - & - & - & + & + & + & + & & - & - & + & - & - & + \\
\hline 5 & + & + & - & - & + & - & - & + & + & - & + & + & - & - & + & - & - & + \\
\hline 6 & + & - & - & + & - & - & + & - & + & - & - & + & + & - & + & - & + & - \\
\hline 7 & - & + & - & + & - & - & + & + & + & + & - & - & + & - & + & - & + & - \\
\hline 8 & - & - & - & + & - & - & + & + & + & - & - & - & - & - & + & - & + & - \\
\hline 9 & - & - & - & - & - & - & + & + & + & - & + & - & - & - & - & + & - & + \\
\hline
\end{tabular}

+ positive; - negative

by Western blot, belonging to a student presenting mild Lyme-related symptoms, and with a history of tick bites. The third C6 positive was negative by lgG Western blot. Four of nine IgM ELISA positive samples were IgG Western blot positive and two had indeterminate results. Ten percent of the positive samples by any ELISA test presented indeterminate results by IgG Western blot. The samples yielding negative in all ELISA tests were not tested by Western blot. Table I shows IgG Western blot positive samples. All of them were positive or in the grey zone by ELISA.

Reactive antibodies to specific antigens were observed. Antibodies to OspA (p31) were found in 89\% of IgG Western blot positive samples, $67 \%$ to p83, and $45 \%$ to $\mathrm{BmpA}$ (p39). In addition, reactivity to nonspecific antigens was observed; that is, $100 \%$ were reactive to heat shock protein p62, 89\% to p57/59 antigen, $67 \%$ to flagellar antigen, $56 \%$ to p75, and $45 \%$ to p50 and p36 (Table II).

\section{Discussion}

A chain of mountains abundant in deer and wild fauna carrying ticks surrounds Monterrey and the neighboring metropolitan area. In the state of Nuevo León there have been identified Ixodes, Rhipicephalus, Dermacentor and Amblyomma [12]. In this environment, it is therefore not surprising that people in contact with ticks have had exposure to B. burgdorferi-infected ticks.

ELISA made with whole-cell antigens presents potential for false positives because nonspecific antibodies can cross-react. The use of recombinant proteins shows high specificity and reduced cross-reactivity of antibodies to Leptospira spp., Brucella spp., and A. marginale and Treponema, as demonstrated in analyses of dog, horse, and human sera [21, 22]. To avoid false po- sitives, the ELISA tests selected for this study contained purified or recombinant antigens.

Nine persons (22.5\%) presented mild clinical symptoms suggestive of Lyme disease; eight of them were negative to antibodies to Borrelia. Eight individuals with antibodies to Borrelia by IgG Western blot were asymptomatic. These data are consistent with findings obtained previously in other countries, demonstrating that workers often exposed to ticks had a high proportion of asymptomatic, positive serologic responses, while the number of clinical cases was relatively low $[23,24]$. There are no data on the real seroprevalence of antibodies to $B$. burgdorferi in asymptomatic or symptomatic people in Mexico.

None of these samples was positive by the three ELISA tests employed. The poor sensitivity of ELISA tests has been documented [5, 23]. Among the factors leading to seronegativity in diagnostic tests there have been identified the wide variety of species and subspecies of Borrelia that co-exist in different parts of the world [1]. The C 6 assay has become the test of choice for the detection of antibodies to $B$. burgdorferi. We found three positive samples by C6 ELISA, but only one was confirmed by IgG Western blot. We could establish that only the C6 positive samples were true positives; however, we cannot exclude the existence of strains with genetic heterogeneity in this region. Nuevo León shares a border with the southern United States. Lin et al. [8] found greater genetic heterogeneity among $B$. burgdorferi sensu lato strains isolated from the southern United States than among strains isolated from the northern United States. Furthermore, they found that the TXW-1 strain isolated from $D$. variabilis ticks appeared to be closer to the European B. garinii genospecies than to other genospecies $[8,25,26]$. It remains to be confirmed whether the TXW-1 strain can be isolated from Lyme 
disease competent vectors. Recently in the USA, Pritt et al. described a new pathogenic B. burgdorferi sensu lato genospecies (candidatus B. mayonii) in the upper Midwestern USA, which causes Lyme borreliosis with unusually high spirochetemia, and distinct clinical features such as symptoms suggesting neurological inclusion and distinct PCR for diagnosis [27].

Furthermore, IgG ELISA and IgM ELISA used in this study included purified or recombinant antigens from B. burgdorferi sensu stricto, B. afzelii and B. garinii; thus we cannot rule out the possibility that ELISA positive samples have antibodies reactive to $B$. afzelii or B. garinii antigens. Escalier and Meza [28] reported acrodermatitis chronica atrophicans (ACA) resistant to multiple treatments in a 69-year-old peasant from South Mexico, bitten by ticks. Lesions improved with doxycycline. Acrodermatitis chronica atrophicans is associated with B. afzelii infection.

The employment of C6 ELISA and European kits COuld be questioned. However, recently Wormser et al. [29] conducted a study to determine whether the tests used in the United States detect Lyme borreliosis acquired in Europe and vice versa. The sensitivity of European and US kits was not similar. In Europe, different diagnostic tests are used for the Borrelia species predominantly found in each given region [30]. The best criterion to diagnose Lyme disease in our country remains to be established, because it is probable that different strains coexist in Mexico, as in the southern United States.

The specific antigenic band most recognized by antibodies in the IgG Western blot was OspA antigen p31 (88.8\%). There have been reported antibodies against OspA antigen during late infection [29-32]; moreover, Malawista et al. [33] reported that spirochetes with mutations in OspA induced strong antibody responses. It is unclear whether the detected frequency of antibodies to OspA in this study indicates that individuals have interacted for a long time with $B$. burgdorferi or perhaps strains circulating in this geographical zone have an immunodominant OspA mutation that elicits frequent antibody responses.

All individuals, with the exception of one with antibodies to Borrelia by IgG Western blot, were asymptomatic. All reported frequent tick exposure and tick bite history, but nobody reported development of erythema migrans. It drew our attention that positive sera samples also shared antibody reactivity to markers of late immune response p83 (67\%) and 45\% to BmpA, even if individuals did not present symptoms of Lyme arthritis or post-Lyme disease characterized by prolonged somatic and neurocognitive symptoms, such as fatigue, difficulty in sleeping, arthralgia, myalgia, memory impairment, and headache [5].
It is unclear whether these findings indicate that individuals have a high susceptibility to develop Lyme disease or perhaps have been in contact for a long time with antigens without developing the disease. Cisak et al. in 2005 and Fahrer et al. in 1991 [29, 30] reported that workers often exposed to ticks had a high proportion of asymptomatic, positive serologic responses, while the number of clinical cases was relatively low. It would be interesting to know the antibody profiles in the populations studied by these groups [24, 25]. Another possibility could be high expression of this antigen by the $B$. burgdorferi sensu stricto strain employed in the Western blot used in this study.

More research is needed to detect the tick-infested areas and the Borrelia strains circulating in Mexico. The data presented here suggest that Lyme borreliosis constitutes a public health threat in Mexico, considered a non-endemic area.

Veterinarians should be informed and alert to take precautionary measures to prevent tick-borne diseases.

\section{Acknowledgments}

This project was partially supported by Universidad Autónoma de Nuevo León; PAICYT-SA 1630-07L, Rheumatology Unit, Medical Faculty Grant and Biotechnology Institute, Faculty of Biological Sciences Grant. We thank Andres Mendiola and Dina Castro Jimenez for their technical support. Dr. Richard G. Robbins, Armed Forces Pest Management Board, Office of the Deputy Under Secretary of Defense for Installations and Environment, Washington, DC, and MC James L. Occi, Emerging Pathogens Department of Medicine, Rutgers Biomedical and Health Science, New Jersey, helpfully reviewed and commented on an earlier version of our manuscript.

The authors declare no conflict of interest.

\section{References}

1. Castro LR, Gabrielli S, Lori A, Cancrini G. Molecular detection of Rickettsia, Borrelia, and Babesia species in Ixodes ricinus sampled in northeastern, central, and insular areas of Italy. Exp Appl Acarol 2015; 66: 443-452.

2. Adelson ME, Rao RV, Tilton RC, et al. Prevalence of Borrelia burgdorferi, Bartonella spp., Babesia microti, and Anaplasma phagocytophila in Ixodes scapularis ticks collected in Northern New Jersey. E J Clin Microbio 2004; 42: 2799-2801.

3. Steere AC, Coburn J, Glickstein L. The emergence of Lyme disease. J Clin Invest 2004; 113: 1093-1101.

4. Aguero-Rosenfeld ME, Wang G, Schwartz I, Wormser GP. Diagnosis of Lyme borreliosis. Clin Microbiol Rev 2005; 18: 484509. 
5. Ścieszka J, Dąbek J, Cieślik P. Post-Lyme disease syndrome. Reumatologia 2015; 53: 46-48.

6. Harvey WT, Salvato P. 'Lyme disease': Ancient engine of an unrecognized borreliosis pandemic? Med Hypotheses 2003; 60: 742-759.

7. Burgess EC, Windberg LA. Borrelia sp. infection in coyotes, black-tailed jack rabbits and desert cottontails in southern Texas. J Wildl Dis 1989; 25: 47-51.

8. Lin T, Oliver JH Jr, Gao L, et al. Genetic heterogeneity of Borrelia burgdorferi sensu lato in the Southern United States based on restriction fragment length polymorphism and sequence analysis. J Clin Microbiol 2001; 39: 2500-2507.

9. Williamson PC, Billingsley PM, Teltow GJ, et al. Borrelia, Ehrlichia, and Rickettsia spp. in ticks removed from persons, Texas, USA. Emerg Infect Dis 2010; 16: 441-446.

10. Cohen ND, Carter CN, Thomas MA Jr, et al. Clinical and epizootiologic characteristics of dogs seropositive for Borrelia burgdorferi in Texas: 110 cases (1988). J Am Vet Med Assoc 1990; 197: 893-898.

11. Mead P, Goel R, Kugeler K. Canine serology as adjunct to human Lyme disease surveillance. Emerg Infect Dis 2011; 17 1710-1712.

12. Guzmán C, Robbins R. The genus Ixodes (Acari: Ixodidae) in Mexico: adult identifcation keys, diagnoses, hosts, and distribution. Rev Mex Biodivers 2010; 81: 289-298.

13. Vargas MH. Lyme disease in Mexico City. Salud Publica Mex 1993; 35: 435-436 [in Spanish].

14. Galaviz-Silva L, Pérez-Treviño KC, Molina-Garza ZJ. Distribution of ixodid ticks on dogs in Nuevo León, Mexico, and their association with Borrelia burgdorferi sensu lato. Exp Appl Acarol 2013; 61: 491-501.

15. Salinas-Meléndez JA, Tamez-González R, Welsh-Lozano O, Barrera-Saldaña HA. Detection of Borrelia burgdorferi DNA in human skin biopsies and dog synovial fluid by the polymerase chain reaction. Rev Latinoam Microbiol 1995; 37: 7-10.

16. Salinas-Meléndez JA, Avalos-Ramírez R, Riojas-Valdez VM, Martínez-Muñoz A. Serological survey of canine borreliosis. Rev Latinoam Microbiol 1999; 41: 1-3.

17. Salinas-Mélendez JA, Galván de la Garza S, Riojas-Valdés VM, et al. Antibody detection against Borrelia burgdorferi in horses located in the suburban areas of Monterrey, Nuevo Leon. Rev Latinoam Microbiol 2001; 43: 161-164.

18. Gordillo-Pérez Torres J, Solórzano-Santos F, et al. Seroepidemiologic study of Lyme's borreliosis in Mexico City and the northeast of the Mexican republic. Salud Publica Mex 2003; 45: 351-355.

19. Gordillo-Pérez G, Torres J, Solórzano-Santos F, et al. Borrelia burgdorferi infection and cutaneous Lyme disease, Mexico. Emerg Infect Dis 2007; 13: 1556-1558.

20. Feria-Arroyo TP, Castro-Arellano I, Gordillo-Perez G, et al. Implications of climate change on distribution of tick vector Ixodes scapularis and risk for Lyme Disease in Texas-Mexico transboundary region. Parasit Vectors 2014; 7: 199.

21. Jackson J, Villarroel A. A survey of the risk of zoonoses for veterinarians. Zoonoses Public Health 2012; 59: 193-201.

22. McDaniel CJ, Cardwell DM, Moeller RB Jr, Gray GC. Humans and cattle: a review of bovine zoonoses. Vector Borne Zoonotic Dis 2014; 14: 11-19.
23. Magnarelli LA, Ijdo JW, Padula SJ, et al. Serologic diagnosis of Lyme borreliosis by using enzyme-linked immunosorbent assays with recombinant antigens. J Clin Microbiol 2000; 38: 1735-1739.

24. Cisak EJ, Chmielewska-Badora J, Zwolinski A, et al. Risk of tickborne bacterial diseases among workers of Roztocze national park (southeastern Poland). Ann Agric Environ Med 2005; 12: 127-132.

25. Fahrer H, Van der Linden SM, Sauvain MJ, et al. The prevalence and incidence of clinical and asymptomatic Lyme borreliosis in a population at risk. J Infect Dis 1991; 163: 305-310.

26. Lin T, Gao L, Seyfang A, Oliver JH Jr. 'Candidatus Borrelia texasensis', from the American dog tick Dermacentor variabilis. Int J Syst Evol Microbiol 2005; 55: 685-693.

27. Pritt BS, Mead PS, Johnson DK, et al. Identification of a novel pathogenic Borrelia species causing Lyme borreliosis with unusually high spirochaetaemia: a descriptive study. Lancet Infect Dis 2016; pii: S1473-3099(15)00464-8.

28. Escalier-Palmer MG, Meza-Nava Al. Borreliosis. A. Clinic case. Rev Sanid Milit Mex 2006; 60: 346-351 [in Spanish].

29. Wormser GP, Tang AT, Schimmoeller NR, et al. Utility of serodiagnostic designed for use in the United States for detection of Lyme borreliosis acquired in Europe and vice versa. Med Microbiol Immunol 2014; 203: 65-71.

30. Kalish RA, Leong JL,Steere AC. Association of treatment-resistant chronic Lyme arthritis with HLA-DR4 and antibody reactivity to Osp A and Osp B of Borrelia burgdorferi. Infect Immun 1993; 61: 2774-2779.

31. Montgomery RR, Malawista SE, Feen KJ, Bockenstedt LK. Direct demonstration of antigenic substitution of Borrelia burgdorferi ex vivo: Exploration of the paradox of the early immune response to outer surface proteins $\mathrm{A}$ and $\mathrm{C}$ in Lyme disease. Exp Med 1996; 183: 261-269.

32. Steere AC, Drouin E E, Glickstein LJ. Relationship between Immunity to Borrelia burgdorferi Outer-surface Protein A (OspA) and Lyme Arthritis. Clin Infect Dis 2011; 52: 259-265.

33. Malawista SE, Montgomery RR, Wang XM, et al. Geographic clustering of an outer surface protein A mutant of Borrelia burgdorferi. Possible implications of multiple variants for Lyme disease persistence. Rheumatology 2000; 39: 537-541. 